\title{
The Effect of Vitamin D on Depression in Individuals
}

\author{
Mohammad Zahedi', Alireza Razavi², Moosa Sajjadi ${ }^{3}$, Amirreza Nasirzadeh $^{4 *}$ \\ ${ }^{1}$ N1Department of Laboratory Sciences, School of Allied Medical Science, Student Research Committee, Mazandaran University of \\ Medical Sciences, Sari, Iran \\ ${ }^{2}$ Student Research Committee, Mazandaran University of Medical Sciences, Sari, Iran \\ ${ }^{3}$ School of Nursing and Midwifery, Social Development and Health, Promotion Research Center, Gonabad University of Medical \\ Sciences, Gonabad, Iran \\ ${ }^{4}$ Student Research Committee, Gonabad University of Medical Sciences, Gonabad, Iran
}

Corresponding Author: Amirreza Nasirzadeh, Student of Basic Sciences in Nursing, Student Research Committee, Gonabad University of Medical Sciences, Gonabad, Iran. Tel: +98-9155332845, Email: nasirzadeharnz@gmail.com

Received May 3, 2019; Accepted August 28, 2019; Online Published September 24, 2019

\begin{abstract}
Depression is considered as a factor causing significant disability, mortality and healthcare costs and The third leading cause of disability which affects about 840 million people around the world. There are some biological, psychological and environmental theories explaining the pathophysiology of depression, though the main reason is still unknown. Vitamin D as a unique neurosteroid hormone may have an important role in the improvement of depression. Although the relationship between vitamin $\mathrm{D}$ levels and depressive symptoms has been explored, the results are inconsistent. Evidences about the relationship between vitamin D deficiency and depression are in conflict. Many studies indicted that, taking vitamin D supplements can be considered as a useful interventions for depressed patients with vitamins deficiency. The current review study suggests that, higher vitamin D intake may be associated with a lower prevalence of depressive symptoms. Neurons and glia in many parts of the brain such as the cingulate cortex and hippocampus have vitamin D receptors. There are different brain process which vitamin D invovlved including; Neuroimmunomodulation, regulation of neurotrophic factors, neuroprotection, neuroplasticity and brain development are kinds of brain processes. This fact demonstrates that vitamin D might be associated with depression and its supplementation might play an significant role in depression and its treatment.

Keywords: Vitamin D, Cholecalciferol, Depression, Treatment, Supplementation

Citation: Zahedi M, Razavi A, Sajjadi M, Nasirzadeh A. The effect of vitamin D on depression in individuals. Int J Med Rev. 2019;6(3):77-80. doi:10.29252/ijmr-060302.
\end{abstract}

\section{Introduction}

Depression is a mental disability contributing to the increased global burden of disease. ${ }^{1}$ For many years, the role of natural dietary for the management of depression has always been discussed. While many studies reported dietary factors involved in the treatment of depression, some scientific flaws made them limited. The top three dietary preparations considered in prior studies, include omega-3 fatty acids, vitamin $\mathrm{D}$, and the vitamin $\mathrm{B}$ complex. ${ }^{2,3}$ Two main Vitamin D come from herbal and animal source. Its plant form namely D2, called ergocalciferol, and is found in plants such as mushrooms and soy milk. The animal form of vitamin D3 is called cholecalciferol, which is 3 times stronger than D2 and is found in raw fish and in mackerel. ${ }^{4,5}$ In the human body, vitamin D precursor converts to active vitamin D3 by being exposed to sunlight to absorb ultraviolet radiation, thus a major part of the body's vitamin $\mathrm{D}$ is produced. ${ }^{2,6}$ The amount of vitamin D intake to meet the daily needs of the body equals $600 \mathrm{IU}$ in children, and $800 \mathrm{UI}$ in adults up to 70 years old. In people with symptoms of vitamin D deficiency, the vitamin intake can increase up to 1000 IU. ${ }^{7,8}$ Recent Statistics show that, nearly one billion people worldwide suffer from vitamin $\mathrm{D}$ deficiency or inadequate levels. Vitamin D deficiency is a common problem that is not limited to a specific geographic or climatic region, and this problem can be observed even in areas with hot and sunny weather where direct sunlight is easily available. ${ }^{9,10}$ Vitamin D deficiency cause a various side effects on the body. The deficiency of vitamin D during infancy and childhood can lead to irreversible physical and mental retardation. Rickets disease is on of the well known side effect of vitamin D deficiency in children. ${ }^{11,12}$ Individuals who suffered from vitamin D deficiency in their childhood, are more likely to show symptoms of hip fractures in their adolescents. ${ }^{13,14}$ Furthermore, depression may cause feeling loneliness and leaving community, which can lead to decreased vitamin $\mathrm{D}$ intake. ${ }^{15}$ investigate the cause and effect relationship of vitamin deficiency and depression to understand whether it lead to depression or it has the opposite effect, have gained significant importance. ${ }^{16}$ In the present study, we focused on the potential role of vitamin $\mathrm{D}$ for prevention and treatment of

Copyright (C) 2019 The Author(s). This is an open-access article distributed under the terms of the Creative Commons Attribution License (http:// creativecommons.org/licenses/by/4.0), which permits unrestricted use, distribution, and reproduction in any medium, provided the original work is properly cited. 
depression. We aimed to investigate the association between vitamin $\mathrm{D}$ deficiency or insufficiency and depression. In addition, we examined the efficacy of vitamin $\mathrm{D}$ in the treatment of depression.

In randomized controlled trials (RCTs) studies, people with depressive symptoms were part of the study population in addition to patients who had a history of coronary infarction, angina pectoris, stroke, or renal stone disease, as well as pregnant or lactating women, smokers, those with liver problems or substance abuse, or having non-normal creatinine concentrations or taking dietary supplements during the last 2 month were excluded. In these articles, the authors reported that vitamin D supplementation was associated with greater improvement in depression measures. There are several RCTs evaluating the effect of vitamin D supplementation in people with seasonal affective disorder, which indicated it has positive effect on this disorder. Table 1 shows data collected through the literature review and calculated using the method described .

Among various review articles applied in this study, RCT articles studied the link between vitamin D and depression. In these articles amount of vitamin $\mathrm{D}$, frequency, mode of delivery, duration, type of vitamin $\mathrm{D}$ and the dose of vitamin $\mathrm{D}$ were checked out. Although, some articles reported a positive relationship between low vitamin $\mathrm{D}$ levels and depression, but some reports have opposite results. ${ }^{16,23,24}$ Fiqure 1 shows the comparison of the searched administration dose of vitamin D in different RCT studies.

To demonstrated depression of participants due to vitamin D deficiency compared to group with normal vitamin D levels, cross-sectional and cohort studies were needed.

The cross-sectional studies, evaluated the rates of depression and vitamin $\mathrm{D}$ in a population at a single point in time to find out whether there was a link between depression and vitamin D levels. The stabilities of the cohort study included, the deletion of interventional factors and the study of cases, in which some of them had the clinical diagnosis of depression. Table 2 shows populations and sample size of cross-sectional and cohort study.

In recent years, several studies have examined the relationship between vitamin $\mathrm{D}$ and depression, which the results of some of them contradicting each other. A study on men older than 60 years, showed that serum levels 25 $(\mathrm{OH}) \mathrm{D}$ had an inverse relationship with depression. ${ }^{27}$ In a study by Black et al results showed that, an increase of $25(\mathrm{OH})$ D concentration could significantly decrease the depression score. It should be emphasized that, in present study, confounding factors such as age, race, body mass index

Table 1. Characteristics of Vitamin D Supplementation in Randomized Controlled Trials Investigating the Effect of Vitamin D Supplementation on Depressive Symptoms

\begin{tabular}{|c|c|c|c|c|c|c|}
\hline Study, Year (Reference) & $\begin{array}{l}\text { Type of } \\
\text { Study }\end{array}$ & $\begin{array}{c}\text { Administration } \\
\text { Dose of Vitamin D }\end{array}$ & $\begin{array}{l}\text { Mode of } \\
\text { Delivery }\end{array}$ & Participants & Frequency & Duration \\
\hline $\begin{array}{l}\text { Mozaffari Khosravi et al, } \\
2012^{19}\end{array}$ & RCT & $\begin{array}{c}150000 \text { or } 300000 \\
\text { IU }\end{array}$ & IM injection & $\begin{array}{c}120 \text { men and women aged } 20 \text { to } 60 \text { years from specialist } \\
\text { clinics of Yazd Shahid Sadoughi University of Medical } \\
\text { Sciences }\end{array}$ & Twice & $\begin{array}{c}3 \text { months } \\
(12 \mathrm{wk})\end{array}$ \\
\hline Sepehrmanesh et al, $2014^{20}$ & RCT & $50 \mathrm{IU}$ & Capsule & 40 patients between 18 and 65 years old in Kashan & Weekly & $8 w k$ \\
\hline Khoraminya et al, $2012^{21}$ & RDBPC & $1500 \mathrm{IU}$ & Capsule & 42 patients between 18 and 65 years old in Tehran & Daily & $8 w k$ \\
\hline Kjaergaard et al, $2012^{22}$ & RCT & $20000 \mathrm{IU}$ & Capsule & Community members with low $25(\mathrm{OH})$ D level in Norway & Weekly & 6 mon \\
\hline Bertone-Johnson et al, $2012^{23}$ & RCT & $400 \mathrm{IU}$ & Not mentioned & Postmenopausal women in US & Daily & $2 y$ \\
\hline Dean et al, $2011^{24}$ & RCT & $5000 \mathrm{IU}$ & Capsule & Healthy volunteers in Australia & Daily & 6 weeks \\
\hline
\end{tabular}

Abbreviation: RDBPC, Randomized double-blind placebo control.

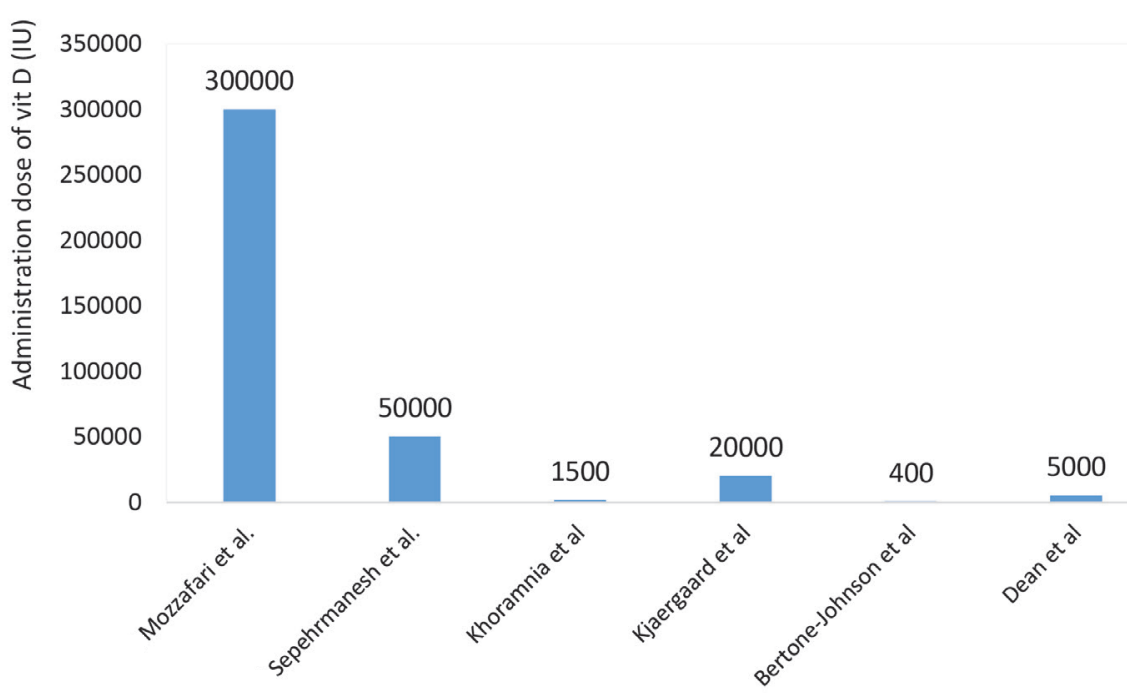

Figure 1. Administration Dose of Vitamin D Per Study. 
Table 2. Study Populations and Sample Size of Cross-Sectional and Cohort Study

\begin{tabular}{|c|c|c|c|}
\hline Study, Year (Reference) & Type of Study & Population & Sample Size \\
\hline Almeida et al, $2015^{25}$ & Retrospective, cross-sectional and prospective & Men aged $71-88$ years who donated a fasting blood sample & 3105 \\
\hline Black et al, $2013^{26}$ & Cross-sectional & Young adult in Australia & 945 \\
\hline Lee et al, $2011^{27}$ & Prospective, non-interventional cohort & Middle-aged and older men & 3369 \\
\hline Milaneschi et al, $2010^{28}$ & Cohort (InCHIANTI Study) & A total of 531 women and 423 men aged $65 \mathrm{yr}$ and older & 954 \\
\hline Milaneschi et al, $2014^{29}$ & Cohort & People between 18 and 65 years old & 2981 \\
\hline Zhao et al, $2010^{30}$ & Cross-sectional & 1890 men and 2026 women & 3916 \\
\hline
\end{tabular}

and physical activity were moderated and controlled. ${ }^{26}$ In another study in the United States, despite the adjustment of confounding factors, there was no significant correlation between vitamin $\mathrm{D}$ deficiency and depression. ${ }^{30}$

The result of study indicated that those who had a low level of $25(\mathrm{OH}) \mathrm{D}$ at baseline had a higher depression score in the following years. It must be pointed out that this finding was more pronounced in women than men. ${ }^{28}$ In a cohort study aimed at determining the relationship between levels of 25 $(\mathrm{OH}) \mathrm{D}$ and depression disorders, the results showed an inverse relationship between the concentration of vitamin $\mathrm{D}$ and the severity of depression symptoms. The strengths of this study included the elimination of confounders and the study of cases in which some of them had the clinical diagnosis of depression. ${ }^{29}$ Almeida et al conducted a study to determine the relationship between the levels of vitamins and incidence of depression in previous, current or future time. The results showed that, vitamin $\mathrm{D}$ concentrations were less than 50 $\mathrm{nmol} / \mathrm{L}$ with a potential for current depression, though it has no effect on past depression. Researchers found that, vitamin $\mathrm{D}$ deficiency was not the cause of depression. ${ }^{30} \mathrm{Li}$ et al analyzed randomized controlled trials, that evaluated the effects of vitamin $\mathrm{D}$ on symptoms of depression. The results showed that, vitamin D supplementation did not have any effect on the reduction of depression, after an intervention. However, they mentioned that, the difference in the duration of the intervention, the dose of vitamin D administered, and the different tools could be the reason for such an outcome. ${ }^{31}$ In another systematic review and meta-analysis study, Spedding examined 15 randomized controlled trials. He concluded that, it would be possible to improve depression symptoms by a daily intake of $800 \mathrm{mg}$ vitamin D. ${ }^{16}$

In a review article by Shaffer et al, the effect of vitamin $\mathrm{D}$ on depression were analyzed. Based on the overall study results, vitamin D had no effect on the improvement of depressed patients. Though, a partial and separate survey of two which was performed on patients with clinically significant depressive symptoms ${ }^{17,19}$ indicated that, vitamin D supplementation intake, had a moderate effect on depressive symptoms. According to the results of mentioned studies, Shaffer et al claimed that, it can be concluded that vitamin $\mathrm{D}$ supplements may be effective in reducing the symptoms of depression in those patients suffering from clinically significant depression. ${ }^{23}$ Mozaffari-Khozravi et al measured the concentration of vitamin D and depression status of the patients and concluded that, vitamin $\mathrm{D}$ had a significant effect on improvement of vitamin D deficiencies.17 Sepehrmanesh et al conducted a study to evaluate the effect of a vitamin supplement on decreasing depression symptoms. Their results showed that, in the vitamin D group, the score for the Beck Depression Inventory (BDI) had significantly decreased. ${ }^{18}$ In regard to relationship between vitamin $\mathrm{D}$ and depression it is important to note that, some studies have not just been conducted on depressed people. Therefore, those who do not have the criteria and characteristics of a depressed person are also included in the study and this has led to errors. Certainly, there are studies that limit this problem and only investigate patients with clinical symptoms of depression, which would report more reliable results. In order to reduce the error rate and increase the accuracy, studies should be conducted on depressed people with vitamin D deficiency in the same age range, who will receive the same amount of vitamin $\mathrm{D}$ using the same method. Furthermore, an outcome evaluation should be carried out with the same tool. Moreover, the previous or current history of taking antidepressants medications should also be considered, since it influences the results, as an intervening variable. Reviewing papers only in the English language, and the necessary inclusion of only published material which may be limited to positive findings are the main limitations of the current analysis, which may risk artificial inflation of results.

\section{Conclusions}

Gratitude to extensive studies in the field of nutrition and neurology, human knowledge has improved regarding the function of the vitamin in the body, especially in the brain. It can be concluded with the high probability that, it is necessary to get the normal amount of vitamin $\mathrm{D}$ needed for normal brain neuropsychiatric function. Many studies show that, deficiency of this particular vitamin increases the risk of depressive disorders. According to the results of the studies, people with high vitamin D deficiency are more likely to develop depression. Moreover, taking vitamin D supplements for those depressed patients who lack vitamins can be considered as useful interventions.

\section{Authors' Contributions}

All authors contributed equally to this study.

\section{Conflict of Interest Disclosures}

The authors declare they have no conflicts of interest. 


\section{Acknowledgments}

The authors would like to appreciate the Student Research Committee and the Gonabad University of Medical Sciences

\section{References}

1. Mohammadi J, Rasoolzadeh Tabatabaei K, Janbozorgi M, Pasandideh A, Salesi M. A Review of Scientific Outputs on Spirituality and Depression Indexed in Important Databases. Int J Med Rev. 2018;5(1):41-46. doi:10.29252/ijmr-050107.

2. Parker GB, Brotchie H, Graham RK. Vitamin D and depression. J Affect Disord. 2017;208:56-61. doi:10.1016/j.jad.2016.08.082.

3. Pu D, Luo J, Wang Y, et al. Prevalence of depression and anxiety in rheumatoid arthritis patients and their associations with serum vitamin D level. Clin Rheumatol. 2018;37(1):179-184. doi:10.1007/s10067-017-3874-4.

4. Ferrari D, Lombardi G, Banfi G. Concerning the vitamin D reference range: pre-analytical and analytical variability of vitamin D measurement. Biochem Med (Zagreb). 2017;27(3):030501. doi:10.11613/bm.2017.030501.

5. Husmann C, Frank M, Schmidt B, et al. Low 25(OH)-vitamin D concentrations are associated with emotional and behavioral problems in German children and adolescents. PLoS One. 2017;12(8):e0183091. doi:10.1371/journal.pone.0183091.

6. May HT, Bair TL, Lappé DL, et al. Association of vitamin D levels with incident depression among a general cardiovascular population. Am Heart J. 2010;159(6):1037-1043. doi:10.1016/j. ahj.2010.03.017.

7. Institute of Medicine Committee to Review Dietary Reference Intakes for Vitamin D, Calcium. The National Academies Collection: Reports funded by National Institutes of Health. In: Ross AC, Taylor CL, Yaktine AL, Del Valle HB, eds. Dietary Reference Intakes for Calcium and Vitamin D. Washington (DC): National Academies Press (US); 2011.

8. de Koning EJ, Lips P, Penninx BW, et al. Vitamin D supplementation for the prevention of depression and poor physical function in older persons: the D-Vitaal study, a randomized clinical trial. Am J Clin Nutr. 2019. doi:10.1093/ajcn/nqz141.

9. Li H, Sun D, Wang A, et al. Serum 25-Hydroxyvitamin D Levels and Depression in Older Adults: A Dose-Response Meta-Analysis of Prospective Cohort Studies. Am J Geriatr Psychiatry. 2019. doi:10.1016/j.jagp.2019.05.022.

10. Berk M, Sanders KM, Pasco JA, et al. Vitamin D deficiency may play a role in depression. Med Hypotheses. 2007;69(6):13161319. doi:10.1016/j.mehy.2007.04.001.

11. Alonso MA, Mantecón L, Santos F. Vitamin D deficiency in children: a challenging diagnosis! Pediatr Res. 2019;85(5):596601. doi:10.1038/s41390-019-0289-8.

12. Mohaddesi H, Saei Ghare Naz M, Najarzadeh M, Yeganehpour M, Khalkhali H. Correlation between Depression with Serum Levels of Vitamin D, Calcium and Magnesium in Women of Reproductive Age. J Caring Sci. 2019;8(2):117-119. doi:10.15171/jcs.2019.017.

13. Holick MF. Vitamin D deficiency. N Engl J Med. 2007;357(3):266281. doi:10.1056/NEJMra070553.

14. Zhu DM, Zhao W, Zhang B, et al. The Relationship Between Serum Concentration of Vitamin D, Total Intracranial Volume, and Severity of Depressive Symptoms in Patients With Major Depressive Disorder. Front Psychiatry. 2019;10:322. doi:10.3389/ fpsyt.2019.00322.

15. Casseb GAS, Kaster MP, Rodrigues ALS. Potential Role of Vitamin D for the Management of Depression and Anxiety. CNS Drugs. 2019;33(7):619-637. doi:10.1007/s40263-019-00640-4.

16. Spedding S. Vitamin D and depression: a systematic review and meta-analysis comparing studies with and without biological flaws. Nutrients. 2014;6(4):1501-1518. doi:10.3390/nu6041501.

17. Shaffer JA, Edmondson D, Wasson LT, et al. Vitamin D supplementation for depressive symptoms: a systematic review and meta-analysis of randomized controlled trials. Psychosom Med. 2014;76(3):190-196. doi:10.1097/psy.0000000000000044.

18. Li G, Mbuagbaw L, Samaan Z, et al. Efficacy of vitamin D supplementation in depression in adults: a systematic review protocol. Syst Rev. 2013;2:64. doi:10.1186/2046-4053-2-64.

19. Mozaffari-Khosravi H, Nabizade L, Yassini-Ardakani SM, Hadinedoushan $H$, Barzegar $K$. The effect of 2 different single injections of high dose of vitamin $\mathrm{D}$ on improving the depression in depressed patients with vitamin D deficiency: a randomized clinical trial. J Clin Psychopharmacol. 2013;33(3):378-385. doi:10.1097/JCP.0b013e31828f619a.

20. Sepehrmanesh Z, Kolahdooz F, Abedi F, et al. Vitamin D Supplementation Affects the Beck Depression Inventory, Insulin Resistance, and Biomarkers of Oxidative Stress in Patients with Major Depressive Disorder: A Randomized, Controlled Clinical Trial. J Nutr. 2016;146(2):243-248. doi:10.3945/jn.115.218883.

21. Khoraminya N, Tehrani-Doost M, Jazayeri S, Hosseini A, Djazayery A. Therapeutic effects of vitamin D as adjunctivetherapy to fluoxetine in patients with major depressive disorder. Aust N Z J Psychiatry. 2013;47(3):271-275.doi:10.1177/0004867412465022.

22. Kjærgaard $M$, Waterloo $K$, Wang CE, et al. Effect of vitamin D supplement on depression scores in people with low levels of serum 25-hydroxyvitamin D: nested case-control study and randomised clinical trial. Br J Psychiatry. 2012;201(5):360-368. doi:10.1192/bjp.bp.111.104349.

23. Bertone-Johnson ER, Powers SI, Spangler L, et al. Vitamin D supplementation and depression in the women's health initiative calcium and vitamin D trial. Am J Epidemiol. 2012;176(1):1-13. doi:10.1093/aje/kwr482.

24. Dean AJ, Bellgrove MA, Hall T, et al. Effects of vitamin D supplementation on cognitive and emotional functioning in young adults--a randomised controlled trial. PLoS One. 2011;6(11):e25966. doi:10.1371/journal.pone.0025966.

25. Almeida OP, Hankey GJ, Yeap BB, Golledge J, Flicker L. Vitamin $\mathrm{D}$ concentration and its association with past, current and future depression in older men: The Health In Men Study. Maturitas. 2015;81(1):36-41. doi:10.1016/j.maturitas.2015.01.016.

26. Black LJ, Jacoby $P$, Allen $K L$, et al. Low vitamin D levels are associated with symptoms of depression in young adult males. Aust N Z J Psychiatry. 2014;48(5):464-471. doi:10.1177/0004867413512383.

27. Lee DM, Tajar A, O'Neill TW, et al. Lower vitamin D levels are associated with depression among community-dwelling European men. J Psychopharmacol. 2011;25(10):1320-1328. doi:10.1177/0269881110379287.

28. MilaneschiY, Shardell M, Corsi AM, et al. Serum 25-hydroxyvitamin $\mathrm{D}$ and depressive symptoms in older women and men. J Clin Endocrinol Metab. 2010;95(7):3225-3233. doi:10.1210/jc.20100347.

29. Milaneschi Y, Hoogendijk W, Lips $P$, et al. The association between low vitamin D and depressive disorders. Mol Psychiatry. 2014;19(4):444-451. doi:10.1038/mp.2013.36.

30. Zhao G, Ford ES, Li C, Balluz LS. No associations between serum concentrations of 25-hydroxyvitamin $\mathrm{D}$ and parathyroid hormone and depression among US adults. Br J Nutr. 2010;104(11):16961702. doi:10.1017/s0007114510002588.

31. Li G, Mbuagbaw L, Samaan Z, et al. Efficacy of vitamin D supplementation in depression in adults: a systematic review. J Clin Endocrinol Metab. 2014;99(3):757-767. doi:10.1210/ jc.2013-3450. 